\title{
Identification of DNA methylation biomarkers with potential to predict response to neoadjuvant chemotherapy in triple-negative breast cancer
}

\author{
Braydon Meyer ${ }^{1}$, Samuel Clifton ${ }^{1}$, Warwick Locke², Phuc-Loi Luu 1,3, Qian Du1,3, Dilys Lam ${ }^{1}$, Nicola J. Armstrong ${ }^{4}$, \\ Beena Kumar ${ }^{5,6,7}$, Niantao Deng ${ }^{3,8}$, Kate Harvey $^{8}$, Alex Swarbrick ${ }^{3,8}$, Vinod Ganju ${ }^{5+}$, Susan J. Clark ${ }^{1,3^{*} \dagger}$, \\ Ruth Pidsley ${ }^{1,3^{*}}$ and Clare Stirzaker ${ }^{1,3^{*}+}$
}

\begin{abstract}
Neoadjuvant chemotherapy (NAC) is used to treat triple-negative breast cancer (TNBC) prior to resection. Biomarkers that accurately predict a patient's response to NAC are needed to individualise therapy and avoid chemotoxicity from unnecessary chemotherapy. We performed whole-genome DNA methylation profiling on diagnostic TNBC biopsy samples from the Sequential Evaluation of Tumours Undergoing Preoperative (SETUP) NAC study. We found 9 significantly differentially methylated regions (DMRs) at diagnosis which were associated with response to NAC. We show that 4 of these DMRs are associated with TNBC overall survival $(P<0.05)$. Our results highlight the potential of DNA methylation biomarkers for predicting NAC response in TNBC.
\end{abstract}

Keywords: DNA methylation, Methylome, Epigenetics, Triple-negative breast cancer, Neoadjuvant chemotherapy

\section{Introduction}

Triple-negative breast cancer (TNBC) represents $\sim 15-20 \%$ of all breast cancers and compared with non-TNBC is associated with a higher risk of disease recurrence after treatment and shorter overall survival [1]. Neoadjuvant chemotherapy (NAC) is typically applied in the TNBC setting, and the degree of pathological response to NAC correlates with long-term prognosis [2]; $30-40 \%$ of TNBC patients achieve a pathological complete response $(\mathrm{pCR})$, associated with a favourable

\footnotetext{
*Correspondence: s.clark@garvan.org.au; r.pidsley@garvan.org.au; c.stirzaker@garvan.org.au

†Vinod Ganju, Susan J. Clark, Ruth Pidsley and Clare Stirzaker: Co-senior authors

${ }^{1}$ Epigenetics Research Laboratory, Genomics and Epigenetics Theme, Garvan Institute of Medical Research, 384 Victoria Street, Darlinghurst, Sydney, NSW 2010, Australia

Full list of author information is available at the end of the article
}

outcome, while patients with a partial or lack of response (non-responder, nR) have a higher risk of relapse and poor prognosis [3]. Biomarkers of NAC response hold the potential to determine which patients will best respond to treatment and identify those patients who will fail to achieve a pCR, allowing personalised chemotherapeutic decision-making approaches within the clinic and avoiding unnecessary toxicity by ineffective treatment. However, there are no reliable methods to date that accurately predict response to NAC.

Epigenetic modifications of tumour DNA, including DNA methylation, are showing widespread promise as molecular biomarkers of disease and treatment response. In this study, we aimed to identify a DNA methylation signature in tumours predictive of response to NAC in TNBC patients.

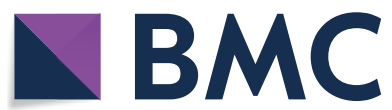

(c) The Author(s) 2021. Open Access This article is licensed under a Creative Commons Attribution 4.0 International License, which permits use, sharing, adaptation, distribution and reproduction in any medium or format, as long as you give appropriate credit to the original author(s) and the source, provide a link to the Creative Commons licence, and indicate if changes were made. The images or other third party material in this article are included in the article's Creative Commons licence, unless indicated otherwise in a credit line to the material. If material is not included in the article's Creative Commons licence and your intended use is not permitted by statutory regulation or exceeds the permitted use, you will need to obtain permission directly from the copyright holder. To view a copy of this licence, visit http://creativecommons.org/licenses/by/4.0/. The Creative Commons Public Domain Dedication waiver (http://creativeco mmons.org/publicdomain/zero/1.0/) applies to the data made available in this article, unless otherwise stated in a credit line to the data. 


\section{Material and methods Clinical samples}

Sequential Evaluation of Tumours Undergoing Preoperative chemotherapy (SETUP) study cancer samples were obtained from Monash and Peninsula Health (Melbourne, Australia). Women with locally advanced TNBC (T1-T3, N0-N3, MO), age > 18, were invited to participate in the SETUP study and were consented for the collection of imaging data and biological specimens for biomarker analysis. Women enrolled in the observational study received NAC with 12 weeks of FEC100 (fluorouracil $500 \mathrm{mg} / \mathrm{m}^{2}$, epirubicin $100 \mathrm{mg} / \mathrm{m}^{2}$, and cyclophosphamide $\left.500 \mathrm{mg} / \mathrm{m}^{2}\right)$, and 12 weeks of docetaxel $\left(100 \mathrm{mg} / \mathrm{m}^{2}\right.$; Fig. 1A). All patients underwent a clinical examination, mammography, ultrasound, breast MRI, and tumour core biopsy at diagnosis (prior to NAC, biopsy A) and all assessments and additional core biopsies were repeated after four cycles of chemotherapy (mid-NAC, biopsy B) and again at completion of the treatment regime (resection, biopsy C) (see Additional file 1 for further study details). Response to NAC was confirmed by PET, CT, and histology, with patients achieving a pathological complete response (pCR) having no evidence of tumour. Patients were considered non-responders $(n R)$ if they showed no evidence of tumour reduction, or a partial responder if their tumour reduced in size but was not entirely eradicated. A total of $n=63$ samples from 32 TNBC patients were acquired for analysis. The SETUP study was approved by the Human Ethics Research Committee and the Monash Medical Centre (ANZCTR.org.au clinical trials identifier: ACTRN12605000588695; HREC/ SETUP/03169A0) as described in Alamgeer et al. [4].

\section{DNA methylation profiling}

DNA was extracted from biopsy samples with the DNeasy kit (Qiagen) as previously described [5]. DNA methylation was quantified using the Illumina Infinium HumanMethylationEPIC BeadChip (Illumina, CA, USA) (EPIC arrays) using the manufacturer's standard protocol. The EPIC array data was processed as previously described [6] (Additional file 1). The resulting dataset comprised 804,805 CpG sites.

Global methylation levels were calculated from repetitive elements targeted by the array using the REMP package (version 1.10.1). Genome-wide differential methylation analysis was performed on biopsies prior to NAC (biopsy A) between patient samples from the extreme ends of response, i.e., those that achieved a pathological complete response ( $\mathrm{pCR}, n=12)$ against those that did not respond to treatment $(\mathrm{nR}, n=7)$. Partial responders $(n=13)$ were excluded from the initial discovery analysis to focus on the extremes of response (pCR and $\mathrm{nR}$ ), as analysis of phenotypic extremes is a common approach in genomic studies to achieve greater statistical power. We included partial responders in secondary analyses to ensure that all treatment responses were considered. We used the limma package to identify differentially methylated probes (DMPs) between these response groups with adjusted $\mathrm{p}$-value cut-off of $\mathrm{FDR}<0.1$. The $\mathrm{R}$ package DMRcate was used to identify differentially methylated regions (DMRs) i.e., response-DMRs, with a DMR p-value cut-off of FDR $<0.1$ and a $\Delta \beta$ of $>=10 \%$ (Additional file 1). $p R O C$ was used for the prediction of response in complete and partial responder samples utilising all significant DMRs.

\section{Survival analysis}

Survival analysis was carried out using Log-rank tests and Cox proportional hazards model as implemented in the $\mathrm{R}$ survival package using overall survival data in the SETUP cohort ( $n=32$ diagnostic biopsies). There were 11 events within this dataset and significance was defined at $P<0.05$. Package details and additional methods for all bioinformatic analysis can be found in Additional file 1.

\section{Results}

To identify if DNA methylation alterations are associated with NAC response, we performed a genome-wide methylation analysis of TNBC samples from the SETUP neoadjuvant clinical study [4]. Biopsies (total $n=63$ ) were

\footnotetext{
(See figure on next page.)

Fig. 1 DNA methylation associated with response to NAC and patient survival in the SETUP study. A Overview of biopsy sample collection in the SETUP study. B Sample availability per patient at diagnosis and mid-NAC for DNA methylation profiling. C Boxplot of tumour purity estimated from DNA methylation in paired samples between biopsies $A$ and $B$, shows that after 12 weeks of treatment complete and partial responders show an average $18 \%(P=0.012)$ and $19.4 \%(P=0.0084)$ reduction in tumour purity respectively. D Dendrogram and heatmap of the 9 significant response-DMRs $(\triangle \beta>10 \%, F D R<0.1)$ found when comparing complete responders $(n=12)$ against non-responders $(n=7)$, with normal breast methylation data shown for reference $(n=4)$. E Receiver operating characteristic (ROC) curve showing the ability of the 9 response-DMRs to distinguish complete $(n=12)$ from partial responders $(n=13)$ on all diagnostic samples (biopsy A, AUC $=0.891)$. F Schematic of the SDR42E1 gene promoter showing location of the response-DMR, individually significant probes and $\beta$ values averaged within response group ( $\mathrm{pCR} n=12$ and $n R n=7$ ). G Boxplot showing significant differential expression of response-DMR SDR42E1 (biopsy A, Welch t-test, $P<0.05$ ). Survival analysis on all 9 response-DMRs was undertaken on the entire SETUP cohort at diagnosis $(n=32)$. $\mathbf{H}$ Kaplan Meier plot of overall survival stratified by patient response (Log-rank test, $P=0.024)$. I Forest plot showing the Cox hazard ratios $( \pm 95 \% \mathrm{Cl})$ for overall survival for each response-DMRs. J Kaplan Meier plot of overall survival for patients in the highest quartile of methylation for response-DMR CDH8 (top 25\%, red) versus the rest of the cohort (bottom 75\%, blue), (HR=1.58 (Cl: 1.06, 2.38), Log-rank test, $P=0.0083)$
} 


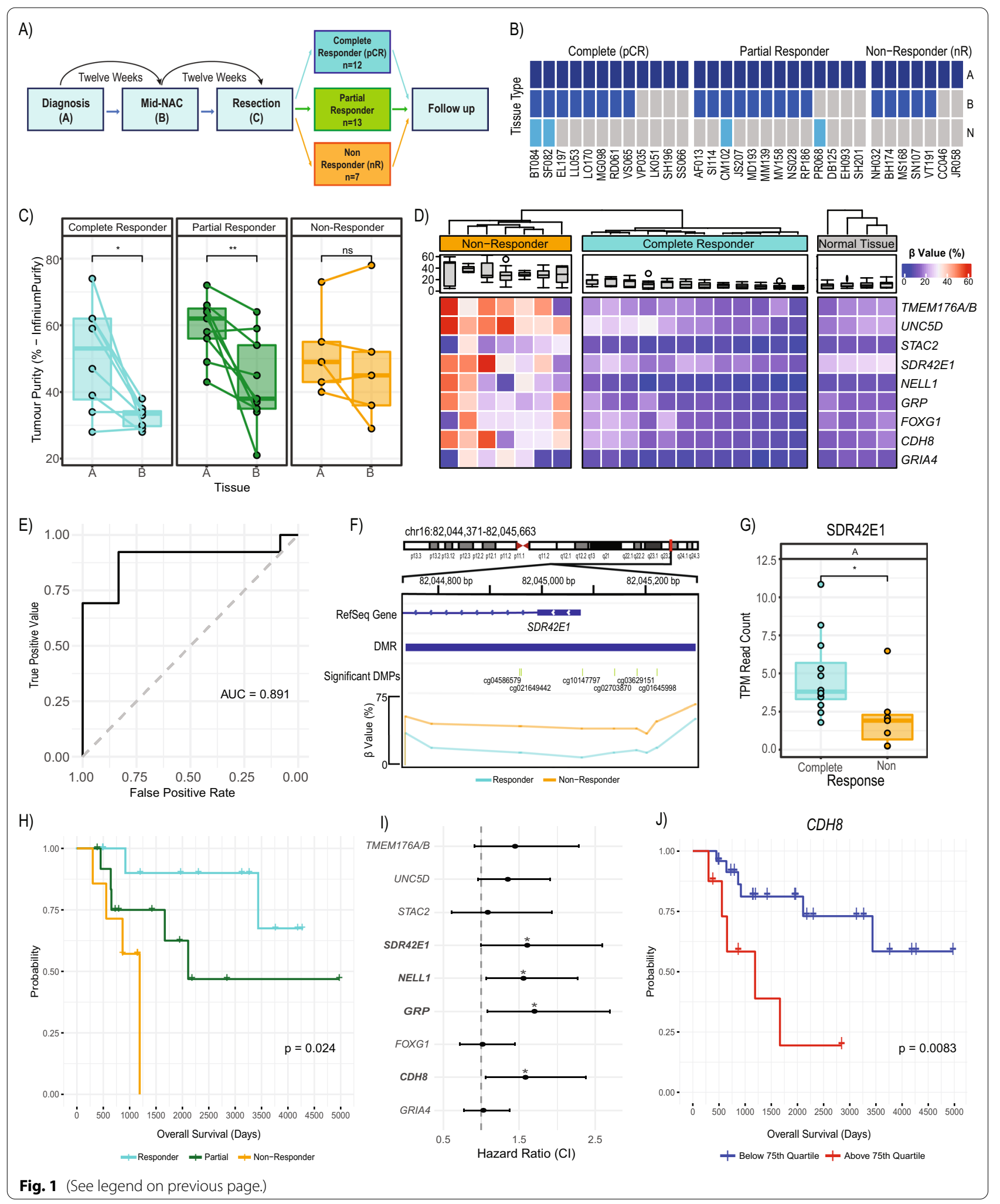


taken at three timepoints: diagnosis (A, $n=32$ tumour, $n=4$ normal adjacent) and mid-chemotherapy (B, $n=22)$ followed by total excision post-chemotherapy $(\mathrm{C}, n=5)$, (Fig. 1A and B). Patients were classified as: pathological complete responders ( $\mathrm{pCR}, n=12)$ where there was no evidence of tumour remaining post-chemotherapy (confirmed by PET, CT, and histology); partial responders $(n=13)$ where there was a reduction in tumour size from baseline; and non-responders $(\mathrm{nR}, n=7)$ where tumour size was unchanged or progressed through chemotherapy [4] (Additional file 3: Table S1 \& S2). We performed DNA methylation profiling of the SETUP biopsy samples using the EPIC arrays, resulting data underwent processing and quality control as detailed in Additional file 1. SNP probes on the array showed that all the samples clustered by patient as expected (Additional file 2: Fig S1).

First, we used cellular deconvolution analyses of the methylation data to quantify tumour purity and celltype composition of each biopsy to inform further analyses. We estimated the epithelial content (representing tumour purity) using two approaches (InfiniumPurify \& EpiDISH, Additional file 1) and found good agreement between the two (Additional file 2: Fig S2a). Whilst there were no significant differences in tumour purity between the responder groups at diagnosis (biopsy A, Additional file 2: Fig S2b), we observed a decrease in tumour purity in response to chemotherapy (biopsy A vs B) in complete $(n=8$ pairs, paired Welch t-test, $P=0.012)$ and partial responders $(n=9$ pairs, paired Welch t-test, $P=0.0084$ ), which was absent in non-responder samples (Fig. 1C). EpiDISH also estimated other cell types including immune and fibroblast contents (Additional file 2: Fig S3). We found a concomitant increase in fibroblast content in those same samples with decreased tumour purity at biopsy B (Additional file 2: Fig S4). No other cell types showed significant changes in abundance with chemotherapy treatment (Additional file 2: Fig S4).

To meet our primary aim of identifying associations between DNA methylation at diagnosis and NAC response, we focused our analysis on patient samples from the extreme end of response, namely $\mathrm{pCR}$ and $\mathrm{nR}$, testing for global, probe-specific, and regional methylation differences. First, we used principal components analysis (PCA) to gain an overview of the association between DNA methylation measurements and known technical and clinical factors (Additional file 1). Each principal component (PC) is linearly independent and explains a particular proportion of the variance within the methylation data. PCs 1 and 2 captured $20.2 \%$ and $13.8 \%$ of the variance respectively and were significantly associated with patient age, EPIC chip, and cellular composition (Pearson correlation, $P=0.1 \times 10^{-5}-9.0 \times 10^{-5}$ ). PC3, capturing $9.6 \%$ of the variation in the data, was significantly and singularly associated with NAC response (Pearson correlation, $P=0.03$, Additional file 2: Fig S5a, b). Global methylation was interrogated through the proxy measure of methylation at targeted repeat regions of the genome including LINE1, Alu and LTR. Using a linear model with PC1 and 2 included as covariates, we observed no significant global methylation differences between responder groups (Additional file 2: Fig S6). Next, we sought to identify probe-specific methylation differences between $\mathrm{pCR}$ and $\mathrm{nR}$ using limma (adjusted for PC1 and 2 above, Additional file 2: Fig S5c, d). We identified 92 significantly differentially methylated probes (DMPs, $F D R<=0.1$, Additional file 3: Table S3), predominantly hypermethylated in non-responders. When hierarchically clustered, these probes show a well-defined discrimination between the two responder groups (Additional file 2: Fig S7a). Inclusion of the partial responders with the same DMPs clusters most partial responders with the non-responder patients (Additional file 2: Fig S7b).

Next, we sought to identify differentially methylated regions (DMRs, co-localised differentially methylated probes) between $\mathrm{pCR}$ and $\mathrm{nR}$ using DMRcate, with DMRs defined as having an absolute methylation difference $(\Delta \beta)$ greater than $10 \%$ and significance of $F D R<=0.1$ (adjusted for PC1 and 2). We identified 9 significant 'response-DMRs', overlapping RefSeq promoters (TMEM176A/B, UNC5D, STAC2, SDR42E1, NELL1, GRP, FOXG1, CDH8, GRIA4) and covering 7-14 CpG sites in each DMR. All 9 DMRs were hypermethylated in $n R$, with a $\Delta \beta$ value (nR-pCR) of $14-28 \%$ (Table 1 , Fig. 1D). We correlated DNA methylation differences in the 9 response-DMRs with tumour purity at diagnosis and found no correlation $(r \leq \pm 0.28, P=\mathrm{ns}$, Additional file 2: Fig S8), confirming that the methylation differences between responder and non-responder samples are independent of tumour purity. We further found no evidence of methylation change at these 9 DMRs before and after NAC (biopsies A vs B), suggesting that their methylation levels are unaffected by treatment (paired t-test, $P>0.05$, Additional file 2: Fig S9). Finally, to ascertain whether the response-DMRs were capable of informing the degree of response to NAC we used receiver operating characteristic (ROC) analysis utilising only the pCR $(n=12)$ and partial responder samples $(n=13)$. Combining all 9 response-DMRs in the ROC analysis we observed an area under the curve of 0.891 , showing that we can also distinguish complete from partial response to NAC with high sensitivity (Fig. 1E).

To determine whether the 9 hypermethylated response-DMRs overlapping gene promoters are associated with gene silencing of these genes in the $n R$ group, we examined RNA-Seq expression data 
Table 1 Details of response-differentially methylated regions (response-DMRs)

\begin{tabular}{lllllllll}
\hline Gene ID & Chr & Range (hg19) & $\begin{array}{l}\text { Average } \\
\Delta \beta(\%)^{\alpha}\end{array}$ & No. CpGs & $P^{\beta}$ & $\begin{array}{l}\text { CpG Island \% } \\
\text { (Island/Shore) }\end{array}$ & $\begin{array}{l}\text { RefSeq \% } \\
\text { (Promoter/ } \\
\text { Exon) }\end{array}$ & $\begin{array}{l}\text { MCF7 ChromHMM \% } \\
\text { (Active/Bivalent/Polycomb/ } \\
\text { Quiescent) }\end{array}$ \\
\hline TMEM176A/B & Chr7 & $150,497,065-150,498,205$ & 27.2 & 14 & $2.04 \times 10^{-9}$ & $100 / 0$ & $90 / 10$ & $0 / 0 / 0 / 100$ \\
UNC5D & Chr8 & $3,509,283-35,093,411$ & 28.2 & 10 & $2.35 \times 10^{-4}$ & $100 / 0$ & $93 / 7$ & $0 / 0 / 100 / 0$ \\
STAC2 & Chr17 & $37,381,830-37,382,301$ & 14.9 & 7 & $3.73 \times 10^{-4}$ & $100 / 0$ & $72 / 28$ & $0 / 0 / 0 / 100$ \\
SDR42E1 & Chr16 & $82,044,738-82,045,297$ & 23.8 & 10 & $1.13 \times 10^{-3}$ & $80 / 20$ & $90 / 10$ & $100 / 0 / 0 / 0$ \\
NELL1 & Chr11 & $20,690,682-20,691,429$ & 22.8 & 12 & $1.49 \times 10^{-3}$ & $100 / 0$ & $83 / 17$ & $0 / 0 / 100 / 0$ \\
GRP & Chr18 & $56,887,002-56,887,785$ & 20.3 & 11 & $2.97 \times 10^{-3}$ & $73 / 27$ & $73 / 27$ & $0 / 0 / 100 / 0$ \\
FOXG1 & Chr14 & $29,235,928-29,236,535$ & 17.5 & 11 & $2.00 \times 10^{-2}$ & $0 / 100$ & $73 / 27$ & $0 / 0 / 100 / 0$ \\
CDH8 & Chr16 & $62,069,806-62,070,365$ & 24.2 & 10 & $2.00 \times 10^{-2}$ & $100 / 0$ & $100 / 0$ & $0 / 10 / 90 / 0$ \\
GRIA4 & Chr11 & $105,480,979-105,481,322$ & 15.4 & 7 & $2.26 \times 10^{-1}$ & $86 / 14$ & $86 / 14$ & $0 / 0 / 100 / 0$ \\
\hline
\end{tabular}

${ }^{a}$ Calculated as Non-responders minus Complete responders

${ }^{\beta}$ Fisher's multiple comparison statistic

${ }^{\gamma}$ Data from ENCODE MCF7 Segmentation (Additional file 1)

previously performed on the same SETUP diagnostic biopsy samples [7] (Additional file 1). We observed that non-responders with a hypermethylated SDR42E1 promoter DMR (Fig. 1F) showed a significant decrease in expression of $S D R 42 E 1$ (Welch t-test, $P=0.025$ ) compared to pCRs (Fig. 1G), with TMEM176A and TMEM176B showing a trend towards significance (Welch t-test, $P=0.054$ and 0.073 respectively). We did not find any significant differences in expression in the other response-DMR genes, indeed the majority showed low levels of expression across all samples (Additional file 3: Table S5), consistent with their respective MCF7 bivalent/repressive ChromHMM states (Table 1) and concordant with TNBC TCGA expression (Additional file 2: Fig S10). We evaluated breast cancer single-cell RNA-seq data $(n=6$ TNBC) [8] and found that the SDR42E1 expression in our diagnostic biopsy samples is likely of epithelial cell origin while the TMEM176A/B expression is likely of stromal origin (Additional file 2: Fig S11).

We next used Cox proportional hazards models to assess the prognostic utility of known clinical variables and methylation in our cohort (partial responders included; $n=32$ ). We show that patient response to NAC is associated with overall survival (Log-rank test, $P=0.024$, Fig. $1 \mathrm{H})[4]$, while other standard clinical details such as age and breast cancer stage did not yield any prognostic utility (Additional file 3: Table S6). We show that average methylation in 4/9 response-DMRs (NELL1, GRP, CDH8, SDR42E1) is significantly associated with overall survival (Cox proportional hazards model, $\mathrm{HR}=1.56-1.70, P<0.05$, Fig. $1 \mathrm{I}$ and J, Additional file 3: Table S7).

\section{Discussion}

The response of a tumour to NAC treatment in TNBC is highly variable and poorly understood. DNA methylation offers great potential as a biomarker of treatment response. Thus, we performed whole-genome methylation profiling in a TNBC NAC cohort to identify novel predictive DNA methylation biomarkers of NAC response.

We identified nine DMRs, significantly hypermethylated in non-responder patient samples, with the ability to distinguish both non-responders and partial responders from complete responders, although this is pending larger cohort validation, which is a limitation of this study. Notably, all nine response-DMR genes have been previously associated with cancer and cancer-related pathways (Additional file 3: Table S4 for details and references). Briefly, hypermethylation of TMEM176A is associated with metastasis and reduced overall survival in colorectal cancer while $U N C 5 D$ is a novel putative metastatic suppressor gene shown to be commonly hypermethylated in prostate cancer. Both STAC2 and NELL1 promoter CpG island hypermethylation has been reported in metastatic breast cancer and primary colon cancer, respectively and GRP is implicated in the development of tumorigenicity and drug resistance. FOXG1 is an evolutionarily conserved forkhead-box transcriptional co-repressor with low levels shown to be associated with poor prognosis in breast cancer. $\mathrm{CDH} 8$ codes for an integral membrane protein from the cadherin family where loss of $\mathrm{CDH} 8$ has been reported in breast carcinoma. GRIA4 hypermethylation has previously been reported to have prognostic value in breast cancer. Interestingly, NELL1 overexpression is associated 
with chemotherapeutic sensitivity to cis/carboplatin and reduced colony formation in lung cancer, suggesting that epigenetic regulation of this gene may be important in chemotherapy response. Finally, SDR42E1 involved in oxidoreductase activity, is the only DMR that shows both altered methylation and expression in this study. Liu et al. has previously shown that SDR42E1 methylation is associated with differential gene expression in TET mutated diffuse large B-cell lymphoma [9], highlighting a potentially important role in cancer and making it a key gene for future functional studies.

Typically, pathological complete response to NAC correlates with better clinical outcomes, while residual disease after NAC is associated with higher risk of relapse and poorer survival among TNBC patients. Indeed, we show that the 9 DMRs associated with TNBC SETUP biopsy response to NAC, also have significant prognostic value in the SETUP patients. Pineda et al. [10] also reported a two gene epigenetic signature (FERD3L and TRIP10) for prediction of response to NAC in TNBC. There is no overlap in the response-DMR biomarkers identified in our two studies, likely due to the discordant neoadjuvant chemotherapy regimens used in each of the discovery cohorts. Nonetheless these studies each demonstrate the value of epigenetic signatures for predicting response to NAC and further exemplify the clinical interest and need for an all-encompassing NAC predictive methylation panel.

Overall, we have shown the potential of DNA methylation to be used as a predictive biomarker of response to NAC in TNBC. The utility of these biomarkers for predicting treatment response and long-term prognostic outcome in TNBC requires validation on larger cohorts and importantly, developing tailor-made biomarkers to specific NAC regimes may be warranted.

\section{Supplementary Information}

The online version contains supplementary material available at https://doi. org/10.1186/s13148-021-01210-6.

Additional file 1. Extended Methods

Additional file 2. Figure S1 Samples cluster by patient according to SNP probes as expected. Non-hierarchical clustering of beta values of 59 SNP quality control probes. Dendrogram coloured by the top 8 clusters found within the data. Figure S2 Tumour purity is not different between responder groups at diagnosis. a) InfiniumPurify and EpiDISH purity estimations are highly correlated (Pearson's correlation) b) Estimated epithelial content for patients grouped by responder status at each timepoint. Dashed line represents median for all samples at each timepoint. Figure S3 All samples exhibit heterogeneous cellular composition. EpiDISH estimated cellular composition of each TNBC sample ( $n=59$, normal samples excluded). Cell types in order of inclusion: Epithelial, natural killer, neutrophils, monocytes, fibroblasts, eosinophils, CD8+T-cells, CD4 + T-cells, and B-cells. Figure S4 Reduction in tumour purity after 12 weeks of treatment in complete and partial responders but not in nonresponders. EpiDISH estimated cellular composition in matched patient biopsy samples at diagnosis (A) and mid-way through NAC (B) (paired t-test). Figure $\mathbf{S} \mathbf{5}$ Variance in DNA methylation at diagnosis associated with response to NAC. Principal components analysis (PCA) shows a) Pearson's correlation (top $=r$, bottom $=P$ ) between $P C$ eigenvector and known clinical and technical variables, and $b$ ) the percentage of variance in the methylation data explained by the top 5 PCs. After identification of PC1 and PC2 as being associated with technical and biological variables other than the variable of interest (response), we demonstrate that after removal of PC1 and PC2 using the removeBatcheffects function, a new $P C$ analysis showed that variation in methylation associated with these variables is removed $(c, d)$. This supports our inclusion of PC1 and PC2 in our linear model to identify response-DMRs. Figure S6 No difference in global methylation between complete responders and non-responders at diagnosis. Global methylation estimated from EPIC array probes overlapping repetitive elements (RE) LINEI, Alu and LTR. Figure S7 Methylation at 92 significant DMPs clusters patients by response to NAC. Dendrogram and heatmap of methylation of the 92 significant DMPs in samples from a) responders $(n=12)$ and non-responders $(n=7)$, and b) with partial responders $(n=13)$ included. Figure S8 Correlation of DNA methylation at response-DMRs with InfiniumPurify derived tumour purity measures. Methylation levels of 9 significant response-DMRs correlated with the InfiniumPurify derived tumour purity shows no correlation between these measures ( $r \leq \pm 0.28, P=n s)$. Points are coloured by response. Figure S9 DNA methylation at response-DMRs is stable between diagnosis and mid-NAC. Methylation levels at the 9 DMRs between biopsies A and B, separated into complete responders and non-responders (paired t-test). Figure $\mathbf{S 1 0}$ Low levels of gene expression at genes with promoter overlapping response-DMRs in the TCGA TNBC dataset. RNA-seq expression levels $\log 2(T P M+1)$. Figure $\mathbf{S 1 1}$ In breast tumour tissue SDR42E1 is localised to the epithelial cells, whilst TMEM176A/B is localised to stromal cells. Single-cell breast cancer data from 6 patients showing expression of the 10 genes proximal to the 9 response-DMRs. Size of dot is proportional to the percentage of cells in that cell type expressing that gene and the colour represents the scaled mean expression of that gene within the cell type

Additional file 3. Supplementary Tables

\section{Acknowledgements}

This work is supported by the National Health and Medical Research Council (NHMRC) project grant (1106870 - SJC, RP) and fellowships (1063559 \& 1156408 - SJC), National Breast Cancer Foundation IIRS Grant (IIRS-18-137 - CS), National Foundation and Medical Research and Innovation grant (NFMRI) (CS), Cancer Council NSW (RG-18-09 - RP, SJC). Computational resources were provided by the Australian Government through NCI Raijin under the National Computational Merit Allocation Scheme 2019, project wk73 (SJC, PLL). Q.D. is a NHMRC Investigator Grant recipient \#1177792. We thank Professor Jane Fox, Director of Breast Services, Department of Surgery, Monash University School of Clinical Sciences at Monash Health for her role in recruiting patients to the study.

\section{Authors' contributions}

RP, CS and SJC coordinated the overall study and wrote the manuscript together with BM. KH prepared DNA for EPIC arrays. BM, PLL, SC, DL, RP, WL, NJA, and ND analysed the data. VG, BK, and AS supplied DNA samples from SETUP study. All authors read and approved the final manuscript.

\section{Availability of data and materials}

The datasets generated and analysed during the current study are publicly available at NCBI GEO (www.ncbi.nlm.nih.gov/geo) under accession numbers GSE184159.

\section{Declarations}

Ethics approval and consent to participate

The SETUP study tumour samples were obtained from Monash and Peninsula Health (Melbourne, Australia). Patients with locally advanced breast cancer, deemed suitable for neoadjuvant cytotoxic chemotherapy, consented for the collection of imaging data and biological specimens for biomarker 
analysis (SETUP study) and were enrolled in a phase III randomised controlled neoadjuvant clinical trial approved by the Human Ethics Research Committee and the Monash Medical Centre (ANZCTR.org.au clinical trials identifier: ACTRN12605000588695; HREC/SETUP/03169A0).

\section{Consent for publication}

Not applicable.

\section{Competing interests}

The authors declare that they have no competing interests.

\section{Author details}

'Epigenetics Research Laboratory, Genomics and Epigenetics Theme, Garvan Institute of Medical Research, 384 Victoria Street, Darlinghurst, Sydney, NSW 2010, Australia. ${ }^{2}$ Molecular Diagnostics Solutions, CSIRO Health and Biosecurity, New South Wales, North Ryde 2113, Australia. ${ }^{3}$ St. Vincent's Clinical School, UNSW Australia, Sydney, NSW 2010, Australia. ${ }^{4}$ Department of Mathematics and Statistics, Curtin University, Perth, WA 6103, Australia. ${ }^{5}$ Hudson Institute of Medical Research, Clayton, VIC 3168, Australia. ${ }^{6}$ Monash Health Pathology, Monash Health, Victoria 3168, Australia. ${ }^{7}$ Monash University, Melbourne, VIC 3168 , Australia. ${ }^{8}$ Cancer Research Theme, Garvan Institute of Medical Research, Sydney, NSW 2010, Australia.

Received: 24 September 2021 Accepted: 7 December 2021

Published online: 18 December 2021

\section{References}

1. Blows FM, Driver KE, Schmidt MK, Broeks A, van Leeuwen FE, Wesseling J, et al. Subtyping of breast cancer by immunohistochemistry to investigate a relationship between subtype and short and long term survival: a collaborative analysis of data for 10,159 cases from 12 studies. PLoS Med. 2010;7(5):e1000279

2. Esserman LJ, Berry DA, Cheang MC, Yau C, Perou CM, Carey L, et al. Chemotherapy response and recurrence-free survival in neoadjuvant breast cancer depends on biomarker profiles: results from the I-SPY 1 TRIAL (CALGB 150007/150012; ACRIN 6657). Breast Cancer Res Treat. 2012;132(3):1049-62.

3. Liedtke C, Mazouni C, Hess KR, André F, Tordai A, Mejia JA, et al. Response to neoadjuvant therapy and long-term survival in patients with triplenegative breast cancer. J Clin Oncol. 2008;26(8):1275-81.

4. Alamgeer M, Ganju V, Kumar B, Fox J, Hart S, White M, et al. Changes in aldehyde dehydrogenase-1 expression during neoadjuvant chemotherapy predict outcome in locally advanced breast cancer. BCR. 2014;16(2):R44

5. Stirzaker C, Zotenko E, Song JZ, Qu W, Nair SS, Locke WJ, et al. Methylome sequencing in triple-negative breast cancer reveals distinct methylation clusters with prognostic value. Nat Commun. 2015;6:5899.

6. Pidsley R, Zotenko E, Peters TJ, Lawrence MG, Risbridger GP, Molloy $P$, et al. Critical evaluation of the Illumina MethylationEPIC BeadChip microarray for whole-genome DNA methylation profiling. Genome Biol. 2016:17(1):208.

7. Brockwell NK, Rautela J, Owen KL, Gearing LJ, Deb S, Harvey K, et al. Tumor inherent interferon regulators as biomarkers of long-term chemotherapeutic response in TNBC. NPJ Precis Oncol. 2019;3:21.

8. Wu SZ, Roden DL, Wang C, Holliday H, Harvey K, Cazet AS, et al. Stromal cell diversity associated with immune evasion in human triple-negative breast cancer. EMBO J. 2020;39(19):e104063.

9. Liu P, Jiang W, Zhao J, Zhang H. Integrated analysis of genomewide gene expression and DNA methylation microarray of diffuse large Bcell lymphoma with TET mutations. Mol Med Rep. 2017;16(4):3777-82.

10. Pineda B, Diaz-Lagares A, Perez-Fidalgo JA, Burgues O, Gonzalez-Barrallo I, Crujeiras $A B$, et al. $A$ two-gene epigenetic signature for the prediction of response to neoadjuvant chemotherapy in triple-negative breast cancer patients. Clin Epigenet. 2019;11(1):33.

\section{Publisher's Note}

Springer Nature remains neutral with regard to jurisdictional claims in published maps and institutional affiliations.

Ready to submit your research? Choose BMC and benefit from:

- fast, convenient online submission

- thorough peer review by experienced researchers in your field

- rapid publication on acceptance

- support for research data, including large and complex data types

- gold Open Access which fosters wider collaboration and increased citations

- maximum visibility for your research: over $100 \mathrm{M}$ website views per year

At BMC, research is always in progress.

Learn more biomedcentral.com/submissions 